\title{
Gesteuerte Geweberegeneration (GTR) - Alternativen zum autologen Knochen?
}

Fischer, K

\begin{abstract}
Aktuell gibt es 2 unterschiedliche Strömungen im Bereich der Knochenersatzmaterialien (KEM): zum einen nicht bzw. sehr langsam resorbierende KEM mit hoher Volumenstabilität (v. a. hochtemperierte bovine KEM) und zum anderen KEM mit höherer Substitutionsrate und ggf. geringerer Volumenstabilität (v. a. allogene KEM). Wird ein parodontaler Defekt mit einem allogenem KEM sowie z. T. auch mit einem xenogenem KEM aufgefüllt, kann dies zur Regeneration führen. In der Parodontologie darf zusätzlich die Kombination mit entsprechenden Membranen bzw. mit Schmelz-Matrix-Proteinen (SMP) nicht unberücksichtigt bleiben, da hierdurch das Regenerationsergebnis signifikant verbessert wird. Bei der klassischen Technik der gesteuerten Geweberegeneration (GTR) kann ggf. ein KEM mit höherer Substitutionsrate (autologe Späne, Allograft, -Tricalciumphosphat) mit einer Membran mit längerer Standzeit (Perikardmembran, kreuzvernetzte Membran) kombiniert werden, um ein stabiles Langzeitergebnis zu erzielen.
\end{abstract}

DOI: https://doi.org/10.1007/s11838-020-00107-z

Posted at the Zurich Open Repository and Archive, University of Zurich

ZORA URL: https://doi.org/10.5167/uzh-194469

Journal Article

Accepted Version

Originally published at:

Fischer, K (2020). Gesteuerte Geweberegeneration (GTR) - Alternativen zum autologen Knochen? Wissen Kompakt, 14(2):69-76.

DOI: https://doi.org/10.1007/s11838-020-00107-z 


\section{Gesteuerte Geweberegeneration (GTR) - wo sind die Alternativen zum autologen Knochen?}

Dr. Kai Fischer

Fachzahnarzt für Parodontologie (ZÄKWL)

Spezialist für Parodontologie (DG Paro)

Abteilung für Zahnerhaltung \& Präventivzahnmedizin, Universität Zürich

Praxis „Zahnspezialisten am Dom“

Kürschnerhof 4

97070 Würzburg

dr.fischer@schuetz-tawassoli.de

\section{Lebenslauf}

2004-2009 Studium der Zahnheilkunde an der Julius-Maximilians-Universität in Würzburg

2009

Staatsexamen

2010-2012 wissenschaftlicher Mitarbeiter, Abteilung für Parodontologie, Universität Würzburg

2011

2013

Promotion

Parodontologie e.V. (DGParo)

2013 - 2014 Honorary Research Associate \& Clinical Teaching Fellow, UCL Eastman Dental Institute, Periodontology Unit, London, UK

2014 - 2016 Oberarzt, Abteilung für Parodontologie, Universität Witten/Herdecke

2017 Fachzahnarzt für Parodontologie der Zahnärztekammer Westfalen-Lippe (ZÄKWL)

seit 2017 Tätigkeit in der Gemeinschaftspraxis „Zahnspezialisten am Dom“, Würzburg

\section{Schlüsselwörter}

gesteuerte Geweberegeneration, Knochenersatzmaterial, Substitutionsrate, Volumenstabilität, Parodontalchirurgie

\section{Zusammenfassung}

\section{Lernziele}

- Welche Unterteilung gibt es mit Blick auf die Herkunft der KEM?

- Worin liegen die Unterschiede zwischen den verschiedenen KEM?

- Was sind die wichtigsten Eigenschaften von KEM?

- Gibt es das „ideale“ KEM für die GTR?

\section{Interessenskonflikt}

Dr. Fischer erhielt Referentenhonorare von Straumann AG, Zimmer Biomet GmbH, Tecnoss Srl 
Blickt man heute auf die Gründe für Zahnextraktionen bei Erwachsenen, so wird man feststellen, dass in den letzten Jahren die Karies an Bedeutung verloren und der Zahnverlust durch entzündlichen Knochen- und Weichgewebsverlust zugenommen hat. Daraus folgt, dass das Fachgebiet der Parodontologie jetzt und in Zukunft eine immer wichtigere Rolle in der täglichen Praxis innehat und haben wird. Neben der antiinfektiösen Therapie und der stringenten Nachsorge im Recallsystem trägt auch die Parodontalchirurgie zum Zahnerhalt bei. Wurde in den Anfängen v.a. die resektive Elimination der pathologischen Taschen mit z.T. erheblichen Verlust an Knochengewebe betrieben, muss das Ziel heute im maximalen Erhalt vorhandener und der Regeneration verlorengegangener Strukturen mittels gesteuerter Geweberegeneration (GTR) sein.

Bereits in den 80er Jahren wurde mit verschiedensten natürlichen und künstlichen Knochenersatzmaterialien versucht Knochendefekte zu regenerieren. Ziel hierbei war es, eine Regeneration und damit Neubildung von Zahnzement, Desmodont und Knochen zu erreichen und keine Reparation über ein langes Saumepithel wie nach Scaling und Wurzelglättung zu erhalten. Der Durchbruch gelang Gottlow et al. (1) durch die Verwendung einer Membran, was die Basis des Konzepts der gesteuerten Geweberegeneration bildet. Die Verwendung von Membranen - heute v.a. resorbierbaren Kollagenmembranen - ermöglicht den Ausschluss der schnellwachsenden Epithelzellen und ermöglicht die Ausbildung neuen parodontalen Gewebes.

\section{Knochenersatzmaterial}

In der Literatur werden neben autologem Knochen verschiedene Knochenersatzmaterialien (KEM) für augmentative Eingriffe im Rahmen der regenerativen Parodontalchirurgie beschrieben. Hierbei werden synthetische von nicht-synthetischen Ersatzmaterialien (allogen, xenogen) unterschieden. Die verschiedenen Materialien unterscheiden sich durch ihre Zusammensetzung und Aufbereitung z.T. stark in ihren Eigenschaften, obwohl das Ursprungsgewebe identisch ist.

Die Anforderungen an ein ideales Knochenaugmentationsmaterial sind:

- hohe Osteoinduktion,

- Osteokonduktion,

- Biokompatibilität,

- Porosität (>300 $\mu \mathrm{m})$,

- Belastungsstabilität,

- Resorbierbarkeit,

- Volumenstabilität,

- Formbarkeit,

- Sterilität,

- Niedrige Patientenmorbidität,

- Lange Lagerungsfähigkeit,

- Vertretbare Produktionskosten,

- Verfügbarkeit.

Im Bereich der oralen Chirurgie und somit auch der Parodontologie wird autologer Knochen nach wie vor als Goldstandard angesehen $(2,3)$. Dieser hat neben einer osteokonduktiven vor allem eine osteoinduktive Wirkung und bietet durch die körpereigene Struktur eine ideale Matrix zur Knochenneubildung und trägt zur parodontalen Regeneration bei. Jedoch hängt die Anzahl der überlebenden Zellen von 
Faktoren wie Operationszeit, Entnahmetechnik und Knochenqualität ab (4). So ist z.B. die Gewinnung mittels Knochenmühle oder Knochenschabern, einer Entnahme mittels Piezochirurgie oder Knochenfiltern vorzuziehen. Autologer Knochen kann entweder lateral des Operationsgebiets oder aus verschiedenen anderen Regionen entnommen werden (z.B. retromolarer Unterkiefer). Dies erfordert aber z.T. ein zusätzliches chirurgisches Eingriffsgebiet und damit gehen intra- sowie postoperative Komplikationen und Risiken wie Verletzungen anatomischer Strukturen sowie eine erhöhte Patientenmorbidität einher (5). Aufgrund der über die Jahre immer kleiner gewordenen Lappentechniken und den damit verbundenen Erfolg (6-8) sowie der relativ kleinen parodontalen Defekte im Vergleich zu prä-implantologischen Augmentationen stellt sich die Frage nach der Notwendigkeit sowie der Sinnhaftigkeit einer ausgedehnten Lappenmobilisation oder Eröffnung eines zweiten Wundbereichs zur Gewinnung von autologen Knochenspähnen.

Um diese Problematik zu umgehen, sind in den letzten Jahren verstärkt KEM mit unterschiedlichen Eigenschaften auf dem Markt erschienen. Hierbei gibt es weltweit regional starke Unterschiede in der Verbreitung der Materialien. Während früher eine geringe Anzahl von KEM primär durch spezialisierte Firmen vertrieben wurden, ist durch die Aufnahme von Biomaterialien in das Produktportfolio der wichtigsten Implantathersteller eine breite Palette an Optionen verfügbar. Hierbei ist es häufig für den Kliniker nicht klar zu erkennen, welche Anforderungen von den verschiedenen KEM erfüllt werden. Alle aktuellen KEM erfüllen oben genannte Anforderungen in unterschiedlichem Ausmaß, wobei eine Osteoinduktivität bis heute nur im geringen Umfang für demineralisierten gefriergetrockneten Spenderknochen (DFDBA) nachgewiesen werden konnte (9).

Weil langfristig eine vollständige knöcherne Regeneration angestrebt ist, sollte das KEM formstabil bleiben und als Leitstruktur dienen, während eine ausreichende Matrix an körpereigenem Knochen ausgebildet wird, die das Volumen des Hartgewebes sichert (10). Nach dem Einbringen vermischt sich das KEM mit Blut und stabilisiert das anschließend entstehende Blutkoagulum innerhalb des Defekts $(11,12)$. Um eine ausreichende Durchsetzung des KEM mit Blut zu gewährleisten, sind eine hohe Porosität und Zwischenräume im Augmentat vorteilhaft. In diese Zwischenräume können Blutgefäße sowie parodontale Ligamentzellen einwandern und die Bildung von neuem Parodontium (Zement, Fasern \& Knochen) einleiten. Erst nach der Ausbildung von Geflechtknochen ist eine Resorption des KEM wünschenswert, um abschließend einen möglichst hohen Anteil an körpereigenem Knochen zu erzielen. Bei einer verfrühten Resorption des KEM und unzureichender Knochenneubildung, kann es zu einem Volumenverlust und der Infiltration mit Bindegewebe statt Hartgewebe kommen. Die Resorptionszeiten bei Knochenersatzmaterialien differenzieren stark und werden in der Literatur mit wenigen bis vielen Monaten angegeben. Einige der KEM werden nur langsam oder gar nicht resorbiert und sind somit nach Jahren noch nachweisbar (13-16). Auch hier stellt sich dem Kliniker die Frage, ob ein vergleichbar kleiner Defekt wie bei der GTR ein KEM mit langer Standzeit erfordert, oder, ob nicht sogar eine lange Standzeit das Ergebnis verfälscht, d.h. kam es zu einer echten Regeneration oder ist der Defekt röntgenologisch nur mit KEM gefüllt bzw. der klinische Attachmentgewinn nur durch das Sondieren auf das langsam resorbierende KEM zu erklären. Gibt es also ein KEM, welches histologisch vorhersagbar zur parodontalen Regeneration beiträgt?

\section{Allogene KEM/Allografts}


Hierbei handelt es sich um Spenderknochen menschlichen Ursprungs (Lebend-, Totspenden; z.B. Maxgraft $囚$, Puros $\AA$, Osteograft $\circledast$ ) und das v.a. in Nordamerika am weitesten verbreitete KEM. Partikuläre Allografts werden beispielsweise im Rahmen eines endoprothetischen Eingriffs gewonnen. Das gewonnene Gewebe wird in verschiedenen Prozessen teils chemisch, physikalisch und thermisch behandelt, aufbereitet und terminal bestrahlt. Nach mehreren Etappen ist der gewonnene Knochen gereinigt, konserviert und sterilisiert. Abhängig vom Aufbereitungsprozess spricht man von "gefriergetrocknetem“ (FDBA), „demineralisiert-gefriergetrockentem“ (DFDBA) oder auch „Lösungsmittel-konserviertem“ KEM. Infektionsübertragungen können durch die strengen Regularien praktisch ausgeschlossen werden - müssen aber aufgeklärt werden - und Fremdkörperreaktionen sind aufgrund der hohe genetische Ähnlichkeit zum Empfänger selten (17). Allogene Materialien werden gut in den neuen Knochen integriert beziehungsweise resorbiert. Der Anteil an neugebildetem, vitalen Knochen ist bei der Anwendung von allogenem KEM meist höher als bei xenogenen Materialien (18). Allogene KEM tragen nachweislich zur Bildung von neuem Attachment bei (19). Sowohl FDBA als auch DFDBA kann erfolgreich mit Schmelz-Matrix-Proteinen (SMP) kombiniert werden und zeigen hier bessere Ergebnisse als die alleinige Anwendung der SMP (20).

Der Vorteil der guten Umsatz- und Knochenneubildungsrate muss abhängig von der klinischen Indikation gegen den Nachteil der gegebenenfalls reduzierten Volumenstabilität abgewogen werden, was bei kleinen parodontalen Defekten eine untergeordnete Rolle spielen wird. Dabei wird FDBA oder rein kortikale Materialien langsamer resorbiert als DFDBA oder rein spongiöse Produkte $(19,21)$.

\section{Xenogene KEM}

- $\quad$ bovin - Rind (BioOss $\AA$, CopiOs $®$, Endobon $®$, Cerabone $\circledR)$

- $\quad$ porcin - Schwein (MP3®, THE Graft巴)

- equin - Pferd (MP3®)

- phykogen - Algen (Frios $\circledR$ )

Hierbei handelt es sich um Knochenersatzmaterial tierischen Ursprungs. Diese KEM werden in unterschiedlichen thermischen und chemischen Verfahren gewaschen, gereinigt und teilweise bestrahlt, um eine mögliche Keimübertragung auf den Empfänger zu verhindern. Weil dies abhängig vom Hersteller meist bei sehr hohen Temperaturen geschieht, verändern einige der KEM ihre physikalischen Eigenschaften. So kann ein bovines KEM nach Aufbereitung analog zu einem Allograft eine hohe Substitutionsrate aufweisen (z.B. CopiOs®) oder durch Hocherhitzung/Sinterung eine sehr geringe oder keine Resorbierbarkeit zeigen (z.B. BioOss $₫$, Cerabone $\left.{ }^{\circledR}\right)$. Die Prozessierung beeinflusst weiterhin z.B. die Oberflächenbeschaffenheit und Hydrophilität. Die Materialien werden in Partikelgrößen zwischen 250 - $2000 \mu \mathrm{m}$ und teilweise mit einem Kollagenanteil bis $20 \%$ angeboten.

Allgemein ist der Anteil der Partikel, die nicht resorbiert werden - abhängig von der Prozessierung -, bei einem Xenograft meist höher als bei einem Allograft (18). Diese verlängerte Volumenstabilität auf Kosten einer geringen Umsatzrate bis hin zur NichtResorbierbarkeit ist je nach Indikation wünschenswert bzw. ein Nachteil bei Augmentationen und Regenerationen. Ein in Bindegewebe integriertes, kaum resorbierbares KEM kann bei der röntgenologischen Verlaufskontrolle so zu einer quasi „falsch-positiven“ Beurteilung führen. Xenogene KEM können zur Bildung von neuem Attachment beitragen (19) und auch Langzeitstudien belegen den klinischen Erfolg im Rahmen der GTR (22). Besonders bovine KEM mit langer Standzeit sind 
wissenschaftlich gut dokumentiert und werden klinisch seit Jahren erfolgreich in Kombination mit Membranen und SMP verwendet.

\section{Synthetische KEM/Alloplasts}

- HA - Hydroxylapatit

- ß-TCP - ß-Trikalziumphosphat

- Kalziumsulfat

Diese umfassen Knochenersatzmaterialien synthetischen Ursprungs. Damit sind die KEM in praktisch unbegrenztem Maß verfügbar und eine Übertragung von möglichen Keimen oder Krankheiten kann ausgeschlossen werden.

Beispiele für alloplastische KEM bestehen meist entweder vollständig aus Hydroxylapatit (HA) oder ß-Tricalciumphosphat (ß-TCP) beziehungsweise aus einer Mischung aus beiden (z.b. BoneCeramic ${ }^{\circledR}$, Osopia $\left.{ }^{\circledR}\right)$. HA ist ein mineralischer Bestandteil von menschlichen Knochen, bei physiologischem pH Wert schwer löslich und wird sehr gut toleriert. Studien zeigen allerdings, dass HA praktisch nicht resorbiert wird und eine fragliche osteokonduktive Wirkung hat (23). Im Gegensatz hierzu resorbiert ß-TCP schneller und mit höherer osteokonduktiver Wirkung (24). Das unlösliche HA dient der Volumenstabilisierung, während ß-TCP resorbiert und durch neugebildeten Knochen ersetzt wird.

In der Literatur finden sich Studien, die zeigen, dass synthetische und xenogene KEM in vergleichbarem Umfang zur Bildung von neuem Knochen führen können $(25,26)$. Im Rahmen der Behandlung von parodontalen Defekten kommt es bei der alleinigen Anwendung von synthetischem KEM häufiger zu einer Reparatur und nicht zu einer Regeneration $(19,27)$ und somit nicht zur Ausbildung von neuem Zement oder einem neuen Faserapparat. In Kombination mit SMP oder Membranen kann jedoch eine erfolgreiche Regeneration parodontalen Gewebes histologisch gefunden werden. Hierbei kann der erreichte Attachmentgewinn vergleichbar zu xenogenem Knochen ausfallen und über Jahre stabil bleiben (22). Umgekehrt scheint kein/kaum zusätzlicher Nutzen eines bi-phasischen Alloplasts bei 2-/3-wandigen Defekten im Vergleich zur alleinigen Anwendung von SMP vorzuliegen bei stabilem Attachmentgewinn über vier Jahr (28).

\section{Fallbericht}

Im vorliegenden Fall soll eine GTR mit porcinem KEM und porciner Perikardmembran an Zahn 43 durchgeführt werden. Im mesialen Aspekt des Zahns zeigte sich eine Sondierungstiefe von $10 \mathrm{~mm}$ bei vorausgegangenem Regenerationsversuch (Abb. 1). Radiologisch waren sowohl vertikale Defekt wie auch einige nicht integrierte KEMPartikel im mesialen Aspekt von Zahn 43 sichtbar (Abb. 2). Nach Lappenpräparation und Darstellung der knöchernen Defektsituation mittels eines Papillenerhaltungslappens sowie einer vertikalen Entlastungsinzision im mesialen Bereich zeigte sich das Ausmaß des Defekts (Abb. 3).

Die nicht knöchern integrierten DBBM-Partikel im apikalen Aspekt wurden entfernt und der Defekt gründlich gereinigt (Abb. 4). Der knöcherne Defekt wurde mit porcinem KEM gefüllt (Abb. 5) und mit einer nativen porcinen Perikard-Membran abgedeckt (Abb. 6). Zur Verbesserung der Wundheilung wurde das Knochenmineral mit Hyaluronsäure angemischt und vor Wundverschluss Hyaluronsäure auf die Perikardmembran appliziert. Der spannungsfreie Wundverschluss erfolgte durch eine tiefe horizontale Matratzennaht und sog. doppelte Einzelknopfnähte (Abb. 7). Bereits bei Nahtentferung 10 Tage post-OP zeigte sich eine stabile reizfreie Gewebemanschette um Zahn 43 (Abb. 8). Im Verlauf der Behandlung wurde eine endodontische Behandlung notwendig. Nach einem Jahr zeigte sich eine Reduktion 
der Sondierungstiefe auf $4 \mathrm{~mm}$ (Abb. 9) sowie eine radiologische zeigte Auffüllung des parodontalen Defektes (Abb. 10).

\section{Fazit für die Praxis}

Aktuell gibt es zwei unterschiedliche Strömungen im Bereich der Knochenersatzmaterialien:

- nicht bzw. sehr langsam resorbierende KEM mit hoher Volumenstabilität (v.a. hochtemperierte bovine KEM),

- KEM mit höherer Substitutionsrate und ggf. geringerer Volumenstabilität (v.a. allogene KEM).

Histologisch kann es durch das Auffüllen eines parodontalen Defekts mit einem allogenem KEM sowie z.T. auch mit einem xenogenem KEM zu einer Regeneration kommen und ist einer alleinigen Lappenoperation überlegen (19). Bei der Verwendung von KEM in der Parodontologie darf zusätzlich die Kombination mit entsprechenden Membranen bzw. mit Schmelz-Matrix-Proteinen nicht unberücksichtigt bleiben, da hierdurch das Regenerationsergebnis signifikant verbessert wird. Bei der klassischen GTR Technik kann ggf. ein KEM mit höherer Substitutionsrate (autologe Spähne, Allograft, ß-TCP) mit einer Membran mit längerer Standzeit (Perikardmembran, kreuzvernetzte Membran) und umgekehrt kombiniert werden - niedrige Subtitutionsrate des KEM bei kurzer Barrierefunktion der Membran - um letztlich ein stabiles Langzeitergebnis zu erzielen.

Leider kann aufgrund der aktuellen Datenlage - es fehlen umfangreiche Vergleichsstudien verschiedener Materialien auch im Bereich der parodontalen Regeneration - keine eindeutige Empfehlung für ein Produkt für alle Defektkonfigurationen gegeben werden. Die wachsende Zahl an Materialien bietet eine große Bandbreite an Korngröße, Resorptionszeit, Applikationsform und Herkunft, sodass Produkte eines Ursprungs durchaus unterschiedliche Ergebnisse liefern können. Allgemein muss festgestellt werden, dass nur wenige Materialien durch klinische Studien gut untersucht und dokumentiert sind, was im starken Kontrast zur Zunahme der prä-klinischen Publikationen zu Biomaterialien in den letzten Jahren steht. Um in Zukunft eine eindeutige Aussage über den Vergleich und die Eignung der großen Vielfalt von KEM für die GTR machen zu können, bedarf es mehr klinischer Studien über einen längeren Beobachtungszeitraum. Außerdem sollten verschiedene Materialkombinationen auf ihre Eignung für verschiedene Defektformen untersucht werden.

Aus Sicht des Autors ist es nicht sinnig ein Material für alle Indikation in der Zahnmedizin zu verwenden, da die Anforderung Defekt- und Patienten-spezifisch verschieden sind. Letztlich ist es das Ziel KEM mit höherer Bioaktivität und einem Gleichgewicht zwischen Resorptionsrate sowie Volumenstabilität für eine ideale Knochenneubildung im Rahmen der parodontalen Regeneration zu finden (29). 


\section{CME Fragen}

1. Was ist das Ziel der Membrananwendung in der GTR?

a) Verhindern des Einwachsens der Epithelzellen [richtig]

b) Ausschluss von Zellen aus dem Parodont

c) Verhindern des Einwachsens von Stammzellen

d) Ausschluss von knochenbildenden Zellen

e) Verhindern des Einwachsens von Blutgefäßen

richtige Antwort: A

2. Sie möchten bei einer 70jährigen Patientin einen regenerativen parodontalchirurgischen Eingriff an Zahn 45 vornehmen. Welches Knochenersatzmaterial verwenden Sie, wenn die Patientin Material mit einem potentiellen Risiko einer Erregerübertragung ablehnt?

a) porcines Knochenersatzmaterial

b) synthetische Knochenersatzmaterial [richtig]

c) allogenes Knochenersatzmaterial

d) equines Knochenersatzmaterial

e) bovines Knochenersatzmaterial

richtige Antwort: B

3. Sie planen bei einem 54järigen Patienten die Behandlung eines parodontalen vertikalen Defektes an Zahn 36. Worüber informieren Sie Ihren Patienten?

a) Der bei der Kombination von synthetischem Knochenersatzmaterial und Membranen erreichte Attachmentgewinn bleibt im Vergleich zu xenogenem Knochen nicht sehr lange stabil.

b) Die Anwendung eines bi-phasischen Alloplasts bringt bei 2-/3-wandigen Defekten im Vergleich zur alleinigen Anwendung von Schmelz-Matrix-Proteinen einen deutlichen zusätzlichen Nutzen.

c) Die in der Literatur vorhandenen Studien zeigen, dass xenogene Knochenersatzmaterialien zu einer größeren Bildung von neuem Knochen führen können synthetische.

d) Bei der alleinigen Anwendung von synthetischem Knochenersatzmaterial kommt es häufiger zu einer Reparatur und nicht zu einer Regeneration. [richtig]

e) Auch bei der Kombination von synthetischem Knochenersatzmaterial mit SchmelzMatrix-Proteinen kann histologisch keine erfolgreiche Regeneration parodontalen Gewebes gefunden werden.

richtige Antwort: D

4. Welche Porosität sollte ein ideales Knochenaugmentationsmaterial besitzen?

a) $>700 \mu \mathrm{m}$ 

b) $>600 \mu \mathrm{m}$
c) $>500 \mu \mathrm{m}$
d) $>400 \mu \mathrm{m}$
e) $>300 \mu \mathrm{m}$ [richtig]

richtige Antwort: E

5. Welches Knochenaugmentationsmaterial wird im Bereich der oralen Chirurgie und auch der Parodontologie als Goldstandard angesehen?
a) bovines Knochenersatzmaterial
b) synthetisches Knochenersatzmaterial
c) autologer Knochen [richtig]
d) gefriergetrocknetes Allograft (FDBA)
e) porcines Knochenersatzmaterial

richtige Antwort: C

6. Sie haben sich mit Ihrem Patienten auf die Verwendung eines synthetischen Knochenersatzmaterials geeinigt. Auf welches Material greifen Sie zurück, wenn eine schnellere Resorption mit höherer osteokonduktiver Wirkung gewünscht wird?
a) Hydroxylapatit
b) Kalziumsulfat
c) Glaskeramik
d) ß-Trikalziumphosphat [richtig]
e) Polylactat

richtige Antwort: D

7. Welches Augmentationsmaterial wählen Sie bevorzugt für ein Indikationsgebiet, bei dem Sie eine hohe Volumenstabilität und somit keine bzw. sehr langsame Resorption benötigen?
a) hochtemperiertes bovines Knochenersatzmaterial [richtig]
b) demineralisierten gefriergetrockneten Spenderknochen
c) ß-Trikalziumphosphat
d) autologen Knochen
e) Kalziumsulfat

richtige Antwort: A

8. In welcher Reihenfolge kann bei alleiniger Anwendung von Knochenersatzmaterial mit einer Regeneration gerechnet werden?

a) autolog $>$ allogen $>$ synthethisch [richtig]

b) synthetisch $>$ autolog $>$ xenogen 
c) xenogen $>$ synthetisch $>$ autolog

d) synthetisch $>$ allogen $>$ autolog

e) xenogen $>$ autolog $>$ allogen

richtige Antwort: A

9. Alle aktuellen Knochenersatzmaterialien erfüllen die im Artikel beschriebenen Anforderungen in unterschiedlichem Ausmaß. Für nur welches Knochenersatzmaterial konnte allerdings bis heute im geringen Umfang eine Osteoinduktivität nachgewiesen werden?

a) In porcinem Knochenersatzmaterial

b) In synthetischem Knochenersatzmaterial

c) In bovinem Knochenersatzmaterial

d) In gefriergetrocknetem Spenderknochen (FDBA)

e) In demineralisiertem gefriergetrockneten Spenderknochen (DFDBA) [richtig]

richtige Antwort: $E$

10. Welches Knochenersatzmaterial zählt zu den Alloplasts?

a) Ersatzmaterial aus Hydroxylapatit [richtig]

b) phykogenes Ersatzmaterial

c) porcines Ersatzmaterial

d) gefriergetrockneter Spenderknochen

e) equines Ersatzmaterial

richtige Antwort: A 


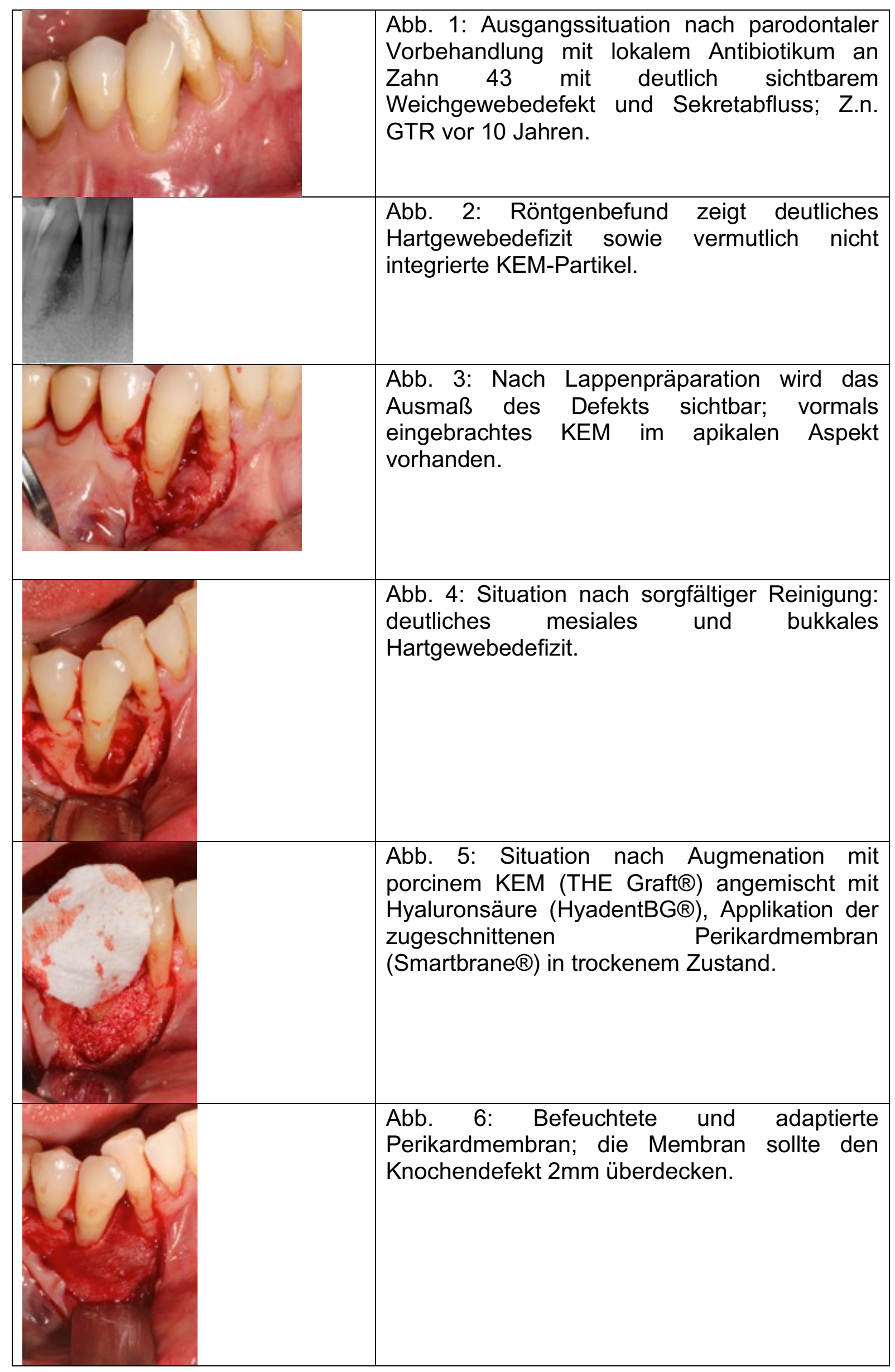




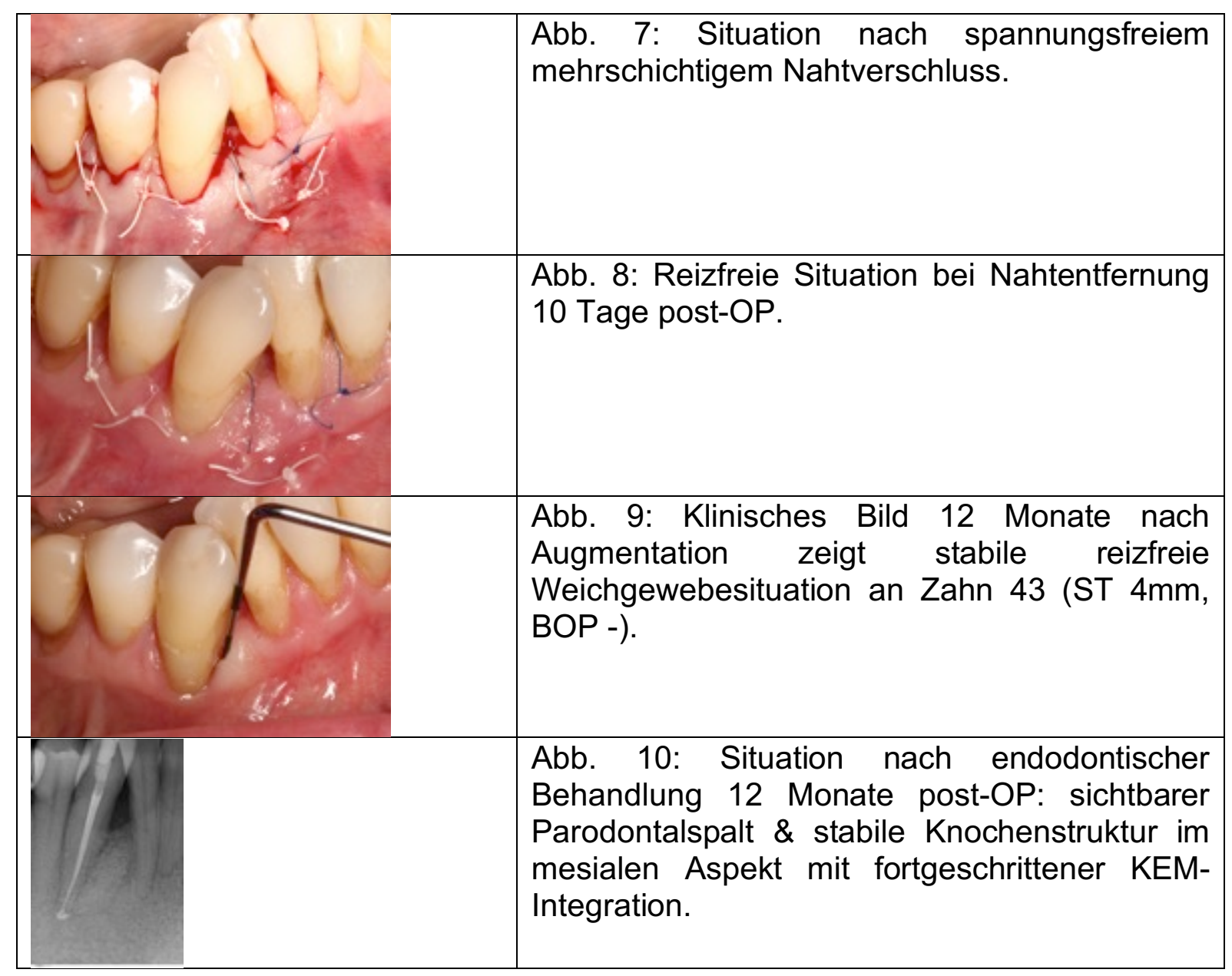




\section{Literatur}

1. Gottlow J, Nyman S, Lindhe J, Karring T, Wennstrom J. New attachment formation in the human periodontium by guided tissue regeneration. Case reports. J Clin Periodontol. 1986;13(6):604-16.

2. Carini F, Longoni S, Amosso E, Paleari J, Carini S, Porcaro G. Bone augmentation with TiMesh. autologous bone versus autologous bone and bone substitutes. A systematic review. Annali di stomatologia. 2014;5(Suppl 2 to No 2):27-36.

3. Klijn RJ, Meijer GJ, Bronkhorst EM, Jansen JA. A meta-analysis of histomorphometric results and graft healing time of various biomaterials compared to autologous bone used as sinus floor augmentation material in humans. Tissue engineering Part B, Reviews. 2010;16(5):493-507.

4. Miron RJ, Gruber R, Hedbom E, Saulacic N, Zhang Y, Sculean A, et al. Impact of bone harvesting techniques on cell viability and the release of growth factors of autografts. Clinical implant dentistry and related research. 2013;15(4):481-9.

5. Nkenke E, Neukam FW. Autogenous bone harvesting and grafting in advanced jaw resorption: morbidity, resorption and implant survival. European journal of oral implantology. 2014;7 Suppl 2:S203-17.

6. Cortellini P, Tonetti MS. Minimally invasive surgical technique and enamel matrix derivative in intra-bony defects. I: Clinical outcomes and morbidity. J Clin Periodontol. 2007;34(12):1082-8.

7. Cortellini P, Prato GP, Tonetti MS. The modified papilla preservation technique. A new surgical approach for interproximal regenerative procedures. J Periodontol. 1995;66(4):261-6.

8. Cortellini P, Prato GP, Tonetti MS. The simplified papilla preservation flap. A novel surgical approach for the management of soft tissues in regenerative procedures. Int $\mathrm{J}$ Periodontics Restorative Dent. 1999;19(6):589-99.

9. Urist MR. Bone: formation by autoinduction. Science. 1965;150(3698):893-9.

10. Carmagnola D, Berglundh T, Lindhe J. The effect of a fibrin glue on the integration of Bio-Oss with bone tissue. A experimental study in labrador dogs. Journal of clinical periodontology. 2002;29(5):377-83.

11. Elgali I, Igawa K, Palmquist A, Lenneras M, Xia W, Choi S, et al. Molecular and structural patterns of bone regeneration in surgically created defects containing bone substitutes. Biomaterials. 2014;35(10):3229-42.

12. Jensen SS, Broggini N, Hjorting-Hansen E, Schenk R, Buser D. Bone healing and graft resorption of autograft, anorganic bovine bone and beta-tricalcium phosphate. A histologic and histomorphometric study in the mandibles of minipigs. Clinical oral implants research. 2006; 17(3):237-43.

13. Bassil J, Naaman N, Lattouf R, Kassis C, Changotade S, Baroukh B, et al. Clinical, histological, and histomorphometrical analysis of maxillary sinus augmentation using inorganic bovine in humans: preliminary results. The Journal of oral implantology. 2013;39(1):73-80.

14. Martinez A, Balboa O, Gasamans I, Otero-Cepeda XL, Guitian F. Deproteinated bovine bone vs. beta-tricalcium phosphate as bone graft substitutes: histomorphometric longitudinal study in the rabbit cranial vault. Clinical oral implants research. 2015;26(6):623-32.

15. Sartori M, Giavaresi G, Tschon M, Martini L, Dolcini L, Fiorini M, et al. Long-term in vivo experimental investigations on magnesium doped hydroxyapatite bone substitutes. Journal of materials science Materials in medicine. 2014;25(6):1495-504.

16. Tete S, Zizzari VL, Vinci R, Zara S, Di Tore U, Manica M, et al. Equine and porcine bone substitutes in maxillary sinus augmentation: a histological and immunohistochemical analysis of VEGF expression. The Journal of craniofacial surgery. 2014;25(3):835-9. 
17. Wang HL, Tsao YP. Histologic evaluation of socket augmentation with mineralized human allograft. The International journal of periodontics $\&$ restorative dentistry. 2008;28(3):231-7.

18. Vance GS, Greenwell H, Miller RL, Hill M, Johnston H, Scheetz JP. Comparison of an allograft in an experimental putty carrier and a bovine-derived xenograft used in ridge preservation: a clinical and histologic study in humans. The International journal of oral \& maxillofacial implants. 2004;19(4):491-7.

19. Reynolds MA, Aichelmann-Reidy ME, Branch-Mays GL, Gunsolley JC. The efficacy of bone replacement grafts in the treatment of periodontal osseous defects. A systematic review. Ann Periodontol. 2003;8(1):227-65.

20. Ogihara S, Tarnow DP. Efficacy of enamel matrix derivative with freeze-dried bone allograft or demineralized freeze-dried bone allograft in intrabony defects: a randomized trial. J Periodontol. 2014;85(10):1351-60.

21. Demetter RS, Calahan BG, Mealey BL. Histologic Evaluation of Wound Healing After Ridge Preservation With Cortical, Cancellous, and Combined Cortico-Cancellous Freeze-Dried Bone Allograft: A Randomized Controlled Clinical Trial. J Periodontol. 2017;88(9):860-8.

22. Dori F, Arweiler NB, Szanto E, Agics A, Gera I, Sculean A. Ten-year results following treatment of intrabony defects with an enamel matrix protein derivative combined with either a natural bone mineral or a beta-tricalcium phosphate. J Periodontol. 2013;84(6):749-57.

23. Stahl SS, Froum SJ. Histologic and clinical responses to porous hydroxylapatite implants in human periodontal defects. Three to twelve months postimplantation. Journal of periodontology. 1987;58(10):689-95.

24. Niedhart C, Maus U, Redmann E, Siebert CH. In vivo testing of a new in situ setting beta-tricalcium phosphate cement for osseous reconstruction. Journal of biomedical materials research. 2001;55(4):530-7.

25. Cordaro L, Bosshardt DD, Palattella P, Rao W, Serino G, Chiapasco M. Maxillary sinus grafting with Bio-Oss or Straumann Bone Ceramic: histomorphometric results from a randomized controlled multicenter clinical trial. Clinical oral implants research. 2008;19(8):796-803.

26. Zafiropoulos GG, Hoffmann O, Kasaj A, Willershausen B, Weiss O, Van Dyke TE. Treatment of intrabony defects using guided tissue regeneration and autogenous spongiosa alone or combined with hydroxyapatite/beta-tricalcium phosphate bone substitute or bovinederived xenograft. Journal of periodontology. 2007;78(11):2216-25.

27. Sculean A, Nikolidakis D, Nikou G, Ivanovic A, Chapple IL, Stavropoulos A. Biomaterials for promoting periodontal regeneration in human intrabony defects: a systematic review. Periodontol 2000. 2015;68(1):182-216.

28. Pietruska M, Pietruski J, Nagy K, Brecx M, Arweiler NB, Sculean A. Four-year results following treatment of intrabony periodontal defects with an enamel matrix derivative alone or combined with a biphasic calcium phosphate. Clinical oral investigations. 2012;16(4):1191-7. 29. Haugen HJ, Lyngstadaas SP, Rossi F, Perale G. Bone grafts: which is the ideal biomaterial? J Clin Periodontol. 2019;46 Suppl 21:92-102. 